\title{
Sinergias universidad y escuela infantil. Aprendizaje-Servicio y el compromiso con los derechos de la infancia
}

\author{
Aurora María Ruiz-Bejarano *
}

Resumen. Durante el curso escolar 2016/2017 la Escuela Infantil Municipal de Primer Ciclo Triquitraque, situada en el municipio gaditano de Puerto Real, inició una serie de actuaciones educativas innovadoras incorporadas a su proyecto educativo. Estas iniciativas perseguían fomentar y consolidar los derechos de la infancia en el seno de su comunidad escolar con la especial implicación de su alumnado. Asimismo, pretendían contribuir al proceso de reconocimiento de la escuela por parte de Unicef como Centro Referente en Educación en Derechos de Infancia y Ciudadanía Global. Profundizando en ambas líneas, se estableció para el curso escolar 2017/2018 la ejecución de una propuesta de colaboración universidad-escuela a través de la asignatura Procesos educativos en Educación Infantil. Por medio de esta iniciativa y como parte de su formación docente, se preveía que una clase de primer curso del Grado en Educación Infantil de la Facultad de Ciencias de la Educación de la Universidad de Cádiz participase en la organización de Una semana por los derechos de la infancia, que se celebró en la Escuela Infantil Municipal Triquitraque entre los días 20 y 24 de noviembre de 2017.

Este artículo analiza la ejecución del proyecto en el marco del aula académica durante los meses de septiembre, octubre y noviembre, así como la participación del alumnado universitario los días 20 y 21 de noviembre en el desarrollo de Una semana por los derechos de la infancia.

Palabras clave: Derechos de la infancia; universidad; escuela; compromiso social; concientización

SINERGIAS ENTRE A UNIVERSIDADE E A ESCOLA DE PRIMEIRA INFÂNCIA. APRENDIZAGEM EM SERVIÇO E O COMPROMISSO COM OS DIREITOS DA CRIANÇA Resumo. Durante o ano letivo de 2016/2017, a Escola Municipal Infantil Triquitraque, localizada no município gaditano de Puerto Real, iniciou uma série de atividades educacionais inovadoras em seu projeto educacional. Essas iniciativas buscavam promover e consolidar os direitos das crianças na comunidade escolar, com o envolvimento especial de seus alunos. Elas também pretendiam contribuir para o processo de reconhecimento da escola pela Unicef como Centro de Referência em Educação em Direitos da Criança e Cidadania Global. Portanto, foi estabelecida para o ano letivo de 2017/2018 a execução de uma proposta de colaboração universidadeescola por meio da disciplina Processos Educacionais na Educação In-

\footnotetext{
* Universidad de Cádiz, España.
} 
fantil. Mediante esta iniciativa e como parte da formação de professores, esperava-se que uma classe do primeiro ano do Curso de Graduação em Educação Infantil da Faculdade de Ciências da Educação da Universidade de Cádiz participasse da organização de Uma semana pelos direitos da criança, que foi realizada na Escola Municipal Infantil Triquitraque entre os dias 20 e 24 de novembro de 2017.

Este artigo analisa a execução do projeto dentro da sala de aula acadêmica durante os meses de setembro, outubro e novembro, bem como a participação de estudantes universitários nos dias 20 e 21 de novembro durante o desenvolvimento de Uma semana pelos direitos da criança.

Palavras-chave: Direitos da criança; universidade; escola compromisso social; conscientização.

SYNERGIES BETWEEN UNIVERSITY AND NURSERY SCHOOL. LEARNING-SERVICE AND THE COMMITMENT TO CHILDREN'S RIGHTS IN

Abstract. During the school year 2016/2017 the First Cycle Nursery School Triquitraque, located in Puerto Real, a village in the province of Cádiz, initiated a series of innovative educational actions implemented in its Educational Programme. These actions aimed to promote and consolidate children's rights in the school community setting with the particular involvement of their pupils. Likewise, they intended to contribute to recognition process of this school as a model in children's rights and global citizenship by UNICEF. After studying both lines in depth, it was established the implementation of a collaborative proposal between the University and the school through the university subject Educational Processes in Infant Education. Through this initiative and as part of their teacher training, it was expected that a group in the first course of the Degree in Infant Education of the Faculty of Education of Cadiz participated in the organization of the Children's Rights Awareness Week, which was held in the Community Nursery School Triquitraque between November 20th and 24th 2017.

This paper analyses both the implementation of the project within the context of the classroom during the months of September, October and November; and the participation of the university students in the development of the Children's Rights Awareness Week, on 20th and 21st November.

Keywords. Children's Rights; university; school; social commitment; awareness.

\section{INTRODUCCIÓN: LA DIMENSIÓN ÉTICA, EL COMPROMISO SOCIAL Y LA RESPONSABILIDAD CÍVICA EN LA UNIVERSIDAD.}

El propósito que orienta este artículo es ofrecer una descripción y un análisis de la estructura organizativa y del proceso de desarrollo seguido en Una semana por los derechos de la infancia, un proyecto llevado a cabo en el curso académico 2017/2018 desde un enfoque de Aprendizaje y Servicio y en el que han participado un grupo universitario de alumnos y de alumnas y una comunidad educativa de una escuela infantil local. 
El proyecto se ha constituido sobre la premisa de que la dimensión ética, la responsabilidad social y el compromiso cívico compartido son tres principios irrenunciables de la formación inicial del profesorado; y en este caso, de la capacitación integral académica de los educadores y las educadoras de educación infantil. En ese sentido, el proyecto Una semana por los derechos de la infancia parte "de una visión de la Universidad como espacio y actor transformador de la sociedad" (Francisco y Moliner, 2010, p. 70). Siguiendo esa línea, una facultad o escuela universitaria de educación insertada en su contexto social, político y cultural e involucrada en su entorno ha de comprometerse en sus aulas con la estimulación de un pensamiento crítico y reflexivo (Schön, 1987; Giroux, 1990) y con la conformación de un ethos socialmente comprometido de su estudiantado que posibilite la generación de procesos de cambio e innovación socioeducativos y favorezca la "transformación de nuestra sociedad en una sociedad más digna, inclusiva, cohesionada y equitativa" (Martínez, 2010, p. 10).

En consonancia con estas ideas, en el dosier resultante de la Conferencia Mundial sobre la Educación Superior. La nueva dinámica de la educación superior y la investigación para el cambio social y el desarrollo, la UNESCO (2009) estimaba que los centros de educación superior han de “promover el pensamiento crítico y la ciudadanía activa, contribuyendo así al desarrollo sostenible, la paz y el bienestar, así como a hacer realidad los derechos humanos, entre ellos la igualdad entre los sexos" (p. 2). De ese modo, la gestación de un pensamiento crítico y de una mirada analítica sobre el entorno social, cultural y educativo se vinculan a la corresponsabilidad ciudadana comprometida dentro de la formación inicial de los educadores y de las educadoras para contribuir a la promoción de una sociedad inclusiva asentada sobre el reconocimiento de los derechos humanos y de los derechos de la infancia y sobre el compromiso con su salvaguarda; una sociedad responsable que propicia un desarrollo sostenible y equilibrado con el medioambiente. Es a lo que Puig, Gijón, Martín y Rubio (2011) se refieren como a la procuración de una "ecoética", pues concierne a:

[...] una ética de la preocupación y del cuidado de la humanidad y de la naturaleza, que resulta totalmente imprescindible en un momento en que la globalización se extiende por todos los ámbitos de la vida y en que la crisis ecológica también se ha generalizado de manera implacable por todos los rincones de la Tierra. Aprender a habitar el mundo es adoptar una ética global y ecológica (p.50)

Una propuesta para un aula de educación superior caracterizada por su concreción a las necesidades de su entorno y por el realismo en sus propósitos y posibilidades puede constituirse, además, en una herramienta 
de empoderamiento para los principales agentes implicados en la iniciativa: el estudiantado universitario y la comunidad educativa de la escuela infantil, en el caso particular del proyecto Una semana por los derechos de la infancia.

El proyecto Una semana por los derechos de la infancia ha sido diseñado desde un enfoque de Aprendizaje y Servicio (en adelante ApS). EI ApS resulta de compleja conceptualización. La literatura especializada ha vertido una multitud de definiciones y la multidisciplinariedad y la multidimensionalidad del ApS se han traducido en una variedad de proyectos y programas de intervención de formas diversas (Martínez-Odría, 2007). Puig, Batlle, Bosch y Palos (2007) sostienen que el ApS es un concepto semejante a un collage, en el sentido de que está dotado de una miríada de elementos característicos que de forma aislada son fáciles de reconocer en otras prácticas y contextos socioeducativos. En función del énfasis puesto en las distintas características, los autores identifican cinco grupos de definiciones aceptadas sobre el enfoque de ApS.

El primer grupo es aquel que concibe el ApS como actividad compleja y de caracterización múltiple que se define por la diferenciación de otras prácticas educativas bien delimitadas. El arraigo de la propuesta de intervención en las necesidades de una comunidad, su cuidada planificación y su estructuración, así como su integración en el currículum establecido, la implicación comprometida del alumnado, su participación activa en el proyecto y el desarrollo y afianzamiento de la responsabilidad ciudadana serían algunas de las características que delimitan el ApS, constituyen su definición y lo diferencian de otras iniciativas educativas.

El segundo grupo pone el énfasis en la caracterización del ApS como programa estructurado. Este reconocimiento del ApS como programa no descuida, sin embargo, dos elementos que le son característicos: su orientación hacia la intervención desde el servicio comunitario y el hecho de que su puesta en marcha conlleva el aprendizaje de destrezas cívicas por parte del alumnado implicado.

El tercer grupo incide especialmente en este último aspecto, en el desarrollo de destrezas y habilidades, concibiendo el ApS como una filosofía o vía para la transformación social y la formación del pensamiento reflexivo y crítico del alumnado que se concreta, a su vez, en una serie de propósitos “1) desarrollar destrezas académicas; 2) formar el pensamiento crítico y reflexivo; 3) afinar la sensibilidad hacia las necesidades de la comunidad; 4) fortalecer el compromiso social; y 5) adquirir actitudes que dispongan a la responsabilidad cívica" (Puig et al., 2007, p. 18). 
El cuarto grupo es aquel conformado por el conjunto de delimitaciones que contemplan el ApS encarándolo principalmente como una forma de pedagogía basada en la experiencia. En este caso, se destaca su vertiente procesual. El ApS se contempla entonces como un dinamismo psicopedagógico de aprendizaje cooperativo que abarca desde el análisis de la situación inicial, la reflexión y la toma de decisiones hasta la evaluación del proyecto sociocultural y educativo y su impacto una vez implementado en la comunidad.

El quinto y último grupo es, según los autores, aquel que apunta más directamente al corazón del ApS en la medida en que, bien sea desde la acentuación del ApS como intervención educativa singular y compleja, como programa estructurado, como filosofía o como pedagogía experiencial, hay una característica invariable en sus conceptualizaciones: el encadenamiento del aprendizaje y del servicio a la comunidad. Batlle (2011) sostiene, precisamente, que la novedad introducida en el ApS no se encuentra en sus componentes (el aprendizaje experiencial y el servicio comunitario), sino en la conjunción de ambos en una misma propuesta. Puig, Gijón, Martín y Rubio (2011), por su parte, apuntan en la misma dirección que Batlle (2011), aunque acentúan el carácter significativo del aprendizaje y mantienen que es en la integración del aprendizaje y de la respuesta a las necesidades de la comunidad donde reside "la novedad y la riqueza del aprendizaje-servicio" (p.52). Aun coincidiendo en el fondo de la cuestión con Batlle (2011) y con Puig et al. (2011), Tapia (2010) se inclina por indicar que son tres los ejes que constituyen el ApS: "el desarrollo de actividades de servicio solidario", "la vinculación intencionada de las prácticas solidarias con los contenidos de aprendizaje y/o investigación incluidos en el currículum" y el "aprendizaje activo" (p. 41). Otras definiciones señalan también hacia esos dos componentes fundamentales del ApS. En primer lugar, "la adquisición de aprendizajes" (Francisco y Moliner, 2010, p. 69) o "el aprendizaje académico" (Martínez-Odría, 2007, p. 630). En segundo lugar, "el servicio voluntario a la comunidad" (Francisco y Moliner, 2010, p. 69) o la "realización de un servicio voluntario a favor de las necesidades detectadas en la comunidad próxima" (Martínez-Odría, 2007, p.630). En una precisa síntesis de todos estos elementos, Puig et al. (2007) aportan una definición de ApS que ha sido ampliamente aceptada y que lo identifica como "propuesta de actividad educativa que combina procesos de aprendizaje y servicio a la comunidad en un solo proyecto bien articulado en el que los participantes aprenden a trabajar sobre necesidades reales del entorno con la finalidad de mejorarlo" (p. 20).

En lo que respecta al contexto universitario, Bisquerra (2008) mantiene que "Ios programas de APS implican a los estudiantes en un servicio a la comunidad bien organizado, pensado para satisfacer necesidades sociales concretas, con la intención de desarrollar aprendizajes académicos, responsabilidad cívica y compromiso comunitario" (p.82). Es decir, dentro del marco 
ofrecido por la educación superior, el ApS integra el servicio dirigido a las necesidades de la comunidad con el aprendizaje del estudiantado y subraya que estos aprendizajes deben incluir la responsabilidad y el compromiso ciudadanos. De esa forma, el ApS posibilita el desarrollo íntegro y entrelazado de las tres dimensiones que conforman una formación universitaria de calidad (Martínez, 2006, 2010). En primer lugar, la dimensión deontológica, referida a los aprendizajes vinculados al ejercicio profesional. En segundo lugar, la dimensión ética y cívica de la enseñanza y el aprendizaje universitario sobre cuya necesaria formación han reparado e insistido diversos autores y autoras (Martínez, Buxarrais y Esteban, 2002; Esteban y Buxarrais, 2004; Bolívar, 2005; Naval, 2010; Buxarrais, Esteban y Mellen, 2015; Repáraz, Arbués, Naval y Ugarte, 2015) y que se halla, además, en sintonía con la misión social de la Universidad descrita por Naval (2010) o con su conceptualización como comunidad ética (Esteban y Martínez, 2012). Y en tercer lugar, la dimensión formativa personal y social que en mayor medida corresponde a niveles educativos inferiores al universitario.

Rodríguez Gallego (2014) apunta que el enfoque de ApS en la educación superior aporta beneficios destacables en tres ámbitos: en el currículum académico, pues permite la proyección y transferencia de los conocimientos teóricos a un plano práctico de necesidades reales e identificables; en el ámbito de la formación en valores, promoviéndose la prosocialidad, el compromiso social y la responsabilidad ciudadana; y en el ámbito de la creación de vínculos con la comunidad, por cuanto el proyecto de intervención surge en el ApS de las necesidades demandadas por un contexto sociocultural específico.

Estas son las coordenadas teóricas que identifican el proyecto Una semana por los derechos de la infanciay que constituyen su matriz. El proyecto se inserta, además, dentro una tradición asentada de ApS en la Facultad de Ciencias de la Educación de la Universidad de Cádiz que arranca en el curso académico 2012/2013 mediante las convocatorias abiertas de innovación y mejora docente (Benítez Gavira, Sánchez Calleja, Zarzuela y García García, 2017) y que se ha ido consolidando a través de un proceso de institucionalización curricular (García García y Cotrina, 2015) y de la creación en el año 2015 de la Asociación de Aprendizaje y Servicio de la Universidad de Cádiz.

\section{LA ESCUELA INFANTIL MUNICIPAL TRIQUITRAQUE}

La Escuela Infantil Triquitraque es un centro educativo municipal de primer ciclo que se encuentra en la ciudad de Puerto Real, a dos kilómetros de distancia de la Facultad de Ciencias de la Educación. En el curso escolar 2017/2018 tiene escolarizados ochenta y dos alumnos y alumnas 
con edades comprendidas entre los cuatro meses y los tres años de edad. Treinta y cuatro de esos niños y niñas están distribuidos en dos aulas de primer año ( $A$ y $B$ ), mientras que los cuarenta y ocho restantes se reparten en tres aulas de segundo año ( $A$-o clase amarilla-, B -o clase verde- y C -0 clase naranja-). La plantilla docente de la escuela infantil está formada por cinco educadoras, un educador y dos auxiliares (mujeres) que rotan entre las distintas aulas para cumplir funciones de apoyo asistencial: cambio de pañales, acompañamiento al comedor, vigilancia en el patio de recreo, etc.

El proyecto educativo de centro de la Escuela Infantil Municipal Triquitraque contempla el compromiso adquirido por toda su comunidad educativa con la Convención de los Derechos del Niño, tratado internacional de las Naciones Unidas aprobado el 20 de noviembre de 1989 en el que por primera vez se reconoce a los niños y a las niñas como sujetos de derechos y que aglutina los derechos políticos, económicos, culturales y sociales de la infancia. Su apuesta por la promoción y la protección de los derechos de la infancia a través de la intervención socioeducativa ha llevado a esta escuela al reconocimiento como Centro Referente en Educación en Derechos de Infancia y Ciudadanía Global en la primera convocatoria suscrita por Unicef en el curso escolar 2016/2017.

Siguiendo las recomendaciones de Unicef, el compromiso de la comunidad educativa con los derechos de la infancia se concreta en su proyecto educativo de centro en cuatro ámbitos. El primer ámbito compete al conocimiento de los derechos de la infancia por parte de las familias, del equipo docente y del propio alumnado. El segundo ámbito refiere a la participación infantil y se orienta hacia la identificación que los alumnos y las alumnas han de hacer de sí mismos y de los demás como sujetos con dignidad, derechos y responsabilidades y como ciudadanos que participan de la vida sociocultural y medioambiental de su entorno. Todo esto sin menoscabo del reconocimiento del valor del juego y del ocio en la infancia. El tercer ámbito se relaciona con la conversión de la escuela infantil en un entorno seguro y promotor de los derechos de la infancia que salvaguarde al alumnado de cualquier forma de discriminación y de violencia física o psicológica. Por último, el cuarto ámbito se concreta en la estimulación de un clima escolar protector de los derechos de la infancia, que garantice el cuidado de la dignidad y de la integridad de todos los miembros de la comunidad educativa, favoreciendo unas relaciones interpersonales respetuosas y una convivencia pacífica en el centro. 


\section{EL PROYECTO UNA SEMANA POR LOS DERECHOS DE LA INFANCIA}

En materia de los derechos de la infancia y en su promoción desde el ámbito educativo, una de las iniciativas aprobadas por la escuela infantil Triquitraque para el curso 2017/2018 fue la organización de Una semana por los derechos de la infancia, tomando como punto de referencia el Día Internacional Del Niño que se celebra cada 20 de noviembre. El proyecto había sido planteado conjuntamente entre la escuela infantil municipal y el grupo $C$ de la asignatura Procesos Educativos en Educación Infantil (perteneciente al primer curso del Grado en Educación infantil), bajo la representación de su profesora responsable ${ }^{1}$.

La propuesta que implicaba Una semana por los derechos de la infancia había partido de las necesidades detectadas por la propia comunidad educativa, así como de las demandas generadas a partir de aquellas necesidades. En primer lugar, la escuela infantil Triquitraque estaba comenzando un nuevo período al adentrarse en el reconocimiento de los derechos de la infancia como una parte irrenunciable de la educación integral de los niños y de las niñas, así como en la incorporación de estos derechos en el proyecto educativo de centro y en su implementación en las intervenciones didácticas de sus aulas y en la dinámica organizativa del centro. En ese sentido, la escuela requería afianzar y solidificar la apuesta que había realizado recientemente por los derechos de la infancia. En segundo lugar, la dirección del centro educativo y el equipo docente buscaban reforzar las relaciones de colaboración y comunicación con las familias del alumnado de la escuela. En tercer lugar y en línea con el punto anterior, se había considerado necesario hacer extensible a las familias la invitación a implicarse y participar activamente en el desarrollo de una educación asentada sobre los derechos de la infancia.

Durante el mes de julio de 2017 y en reuniones mantenidas entre la directora de la escuela infantil y la docente responsable de la asignatura universitaria se acordó, con la aprobación del equipo docente, que la programación de Una semana por los derechos de la infancia contemplaría entre los días 20 y 24 de noviembre de 2017 el desarrollo de talleres didácticos en las aulas del centro y en su salón de psicomotriciad. El tema central de la serie de talleres que los integraría y les aportaría coherencia lo ofrecía Unicef y se trató finalmente del cambio climático y de la educación medioambiental. El salón de psicomotricidad acogería cada mañana un taller intergeneracional en el que participarían el alumnado de una de las clases junto a sus abuelos y abuelas. Los restantes alumnos y alumnas de la escuela llevarían a cabo el

\footnotetext{
${ }^{1}$ Quien suscribe este artículo.
} 
taller didáctico en su aula de referencia. De esta forma, al concluir la semana cada clase habría realizado cuatro talleres didácticos en su aula y un quinto taller en el salón de psicomotricidad con sus abuelos y abuelas.

\subsection{Delimitando los objetivos del proyecto}

Los objetivos que se proponía Una semana por los derechos de la infancia fueron planteados partiendo de la premisa de que el trabajo coordinado y cooperativo entre la escuela infantil y la universidad reporta beneficios valiosos a ambas comunidades (Mendia Gallardo, 2009). Por ese motivo, se plantearon objetivos para los contextos universitario y escolar diferenciadamente y objetivos comunes para ambos contextos.

- Objetivos dentro del marco de la comunidad educativa Triquitraque

- Propiciar en el alumnado de educación infantil la conciencia progresiva del entorno como espacio vital y existencial propio.

- Iniciar al alumnado de educación infantil en la participación activa y cooperativa dentro del marco del cuidado y el respeto al medio ambiente.

- Dar a conocer a las familias el compromiso de la escuela con los derechos de la infancia que se recoge y materializa en el proyecto educativo de centro.

- Implicar conjuntamente a las familias y al profesorado en la promoción de los derechos de la infancia.

- Objetivos dentro el marco universitario

- Favorecer la transferencia de los conocimientos didácticos vinculados a la asignatura Procesos Educativos en Educación Infantil: el sentido de la educación infantil, los procesos de enseñanza y aprendizaje en el aula infantil y la planificación didáctica.

- Afianzar el compromiso ético y la responsabilidad social y ciudadana como canales para la formación de docentes críticos y reflexivos, comprometidos con su entorno educativo, cultural y medioambiental.

- Objetivos comunes: universidad y escuela

- Conocer y comprender la Convención de los Derechos del Niño (1989) y profundizar en el conocimiento de los derechos de la infancia. 


\section{PROMOVER LA SENSIBILIZACIÓN HACIA LOS DERECHOS DE LA INFANCIA Y EL COMPROMISO CON ELLOS. EL CAMBIO CLIMÁTICO EN EDUCACIÓN INFANTIL DESDE LOS DERECHOS DE LA INFANCIA}

A primera vista, el tratamiento del cambio climático en las aulas del primer ciclo de educación infantil aparenta cierta dificultad si se plantea desde un enfoque directo, debido a la complejidad que reviste su imaginario. Conceptos como "calentamiento global" o "efecto invernadero" resultan inaccesibles a los niños y a las niñas de esta etapa educativa. Es posible, sin embargo, realizar una aproximación indirecta al cambio climático que incida, además, en la iniciación a la participación cívica, responsable y comprometida del alumnado de educación infantil con su entorno inmediato, lo que supondría un planteamiento del cambio climático desde el punto de vista de los derechos de la infancia. El núcleo de este enfoque se sitúa en la perspectiva que ofrece La Carta de la Tierra (2000). Su preámbulo realiza la siguiente exposición:

La Tierra, nuestro hogar, está viva con una comunidad singular de vida [...]. La capacidad de recuperación de la comunidad de vida y el bienestar de la humanidad dependen de la preservación de una biosfera saludable, con todos sus sistemas ecológicos, una rica variedad de plantas y animales, tierras fértiles, aguas puras y aire limpio. El medio ambiente global, con sus recursos finitos, es una preocupación común para todos los pueblos (p.1).

El Decreto 428/2008, de 29 de julio, por el que se establece la ordenación y las enseñanzas correspondientes a la Educación Infantil en Andalucía recoge en su tercer artículo que "la finalidad de la educación infantil es la de contribuir al desarrollo físico, afectivo, social e intelectual de los niños y niñas, respetando los derechos de la infancia y atendiendo a su bienestar" (p. 8). Desde esta perspectiva, el entorno próximo o inmediato del niño y de la niña (la escuela, el hogar, el barrio, etc.) adquiere un destacado protagonismo en la educación infantil. Es en este espacio privilegiado de la existencia cotidiana donde tiene lugar, a través de la experimentación, del juego y de las relaciones interactivas y dialógicas con el grupo de iguales o con el adulto, los procesos de enseñanza y aprendizaje, el progresivo desarrollo, la subjetivación y la socialización. De este modo, el espacio existencial de la infancia no es exclusivamente un objeto de la planificación docente, ni constituye solo el campo de las interacciones de los niños y de las niñas con los adultos en contextos extraescolares.

Desde un planteamiento educativo que contemple los derechos de la infancia, comprender el planeta como comunidad de vida y espacio existencial cuya preservación y cuidado constituye una responsabilidad compartida por todos los pueblos y considerar, al mismo tiempo, que es en este espacio de 
posibilidad de la existencia donde se produce el desarrollo integral de los niños y de las niñas conlleva la asunción de estos como sujetos de derechos y ciudadanos activos comprometidos responsablemente con la preservación sostenible de su entorno y con la promoción de una forma de vida equitativa, solidaria, tolerante y pacífica. Bajo esta orientación y estos principios fueron creados los talleres didácticos dentro del proyecto Una semana por los derechos de la infancia.

\section{LA ORGANIZACIÓN DEL GRUPO CLASE UNIVERSITARIO}

El grupo $C$ del primer curso del Grado en Educación Infantil está formado por sesenta y ocho estudiantes. Durante el desarrollo de las clases prácticas, el alumnado de la Facultad de Ciencias de la Educación es repartido en dos subgrupos. En el caso del grupo $C$ de la asignatura Procesos Educativos en Educación Infantil, el Subgrupo / tiene asignadas sus clases prácticas el lunes de cada semana. El Subgrupo /I, por su parte, ocupa una franja del horario de la mañana del martes al desarrollo práctico de esta asignatura.

Para la organización de los talleres didácticos se crearon diez grupos de trabajo: cinco de esos grupos correspondían al Subgrupo / y los cinco restantes al Subgrupo //. El número máximo de estudiantes permitidos en cada grupo era de siete personas. Coincidiendo con las clases prácticas de Procesos Educativos en Educación Infantil, los días en los que el alumnado sería responsable de la organización de los talleres educativos en la escuela infantil serían el lunes 20 y el martes 21 de noviembre.

\section{LAS FASES DE IMPLEMENTACIÓN DEL PROYECTO}

Dentro del marco del trabajo en el aula universitaria, el proyecto se ha ido desarrollando a través de cuatro fases. El grueso de la primera fase consistió en la realización de observaciones dentro de la escuela infantil, tanto en las aulas como en el salón de psicomotricidad y en el patio de recreo. La segunda fase tuvo lugar a lo largo de la organización y preparación de los talleres didácticos dentro del aula universitaria. La tercera fase comprendió los días 20 y 21 de noviembre de 2017, jornadas en las que se ejecutaron los talleres didácticos en la escuela infantil Triquitraque. Y la cuarta y última fase, por su parte, se relacionaba con la evaluación del proyecto por parte del equipo docente del centro de educación infantil y del propio alumnado universitario de la asignatura Procesos educativos en Educación Infantil. 


\subsection{Fase primera: las observaciones en el aula}

La primera de estas fases abarcó el periodo comprendido entre los días 3 y 24 de octubre de 2017 y a lo largo de cinco sesiones ${ }^{2}$ de treinta minutos que se desarrollaron en el interior de la escuela. Su propósito era el conocimiento del grupo-clase donde se llevaría a cabo cada taller mediante la observación en el aula de referencia y en otros espacios de la escuela infantil, así como a través de las conversaciones informales con el educador o la educadora del aula asignada. De manera secundaria y vinculado a este propósito existía otro objetivo de relevancia y profundidad en esta fase: entablar y afianzar en el alumnado el reconocimiento de la necesidad de contextualizar la intervención educativa partiendo de las características individuales y socioculturales del alumnado de educación infantil, de sus necesidades concretas e intereses y de sus aprendizajes previos.

Durante esta primera fase, una pareja de cada grupo de trabajo visitó la escuela infantil. Previamente, en la clase universitaria se había efectuado un debate sencillo acerca de los focos de observación a los que se había de prestar atención durante la estancia en el aula. Sin embargo, esta primera toma de contacto con la escuela y con las aulas de educación infantil se realizó sin el auxilio de un protocolo de observación que orientara la mirada del alumnado dentro de la clase. Con este procedimiento se pretendía que la valoración de los limitados informes resultantes de las observaciones llevara a reflexionar en la clase universitaria acerca de la necesidad de crear protocolos o guiones de observación desde los grupos de trabajo para dirigir una mirada más profunda en el aula de educación infantil. El alumnado fue provisto entonces de un dosier, dotado de unas pautas para la creación de un protocolo de observación, así como de una serie de ejemplos en los que se podía apoyar para construir su propia herramienta de observación.

\subsection{Fase segunda: El diseño y la creación de los talleres}

En una segunda fase se procedió a la toma de decisiones acerca de la estructura y la organización de los talleres didácticos. La intervención del alumnado en la escuela infantil durante la semana por los derechos de la Infancia se prolongaría desde las diez hasta las once y media de la mañana, en tres secuencias de media hora cada una. En primer lugar, se realizaría el desayuno del alumnado de la escuela infantil. Posteriormente, se llevaría a cabo la asamblea de aula. Y finalmente, se ejecutaría el taller didáctico. Los estudiantes universitarios disponían, por tanto, de una hora de intervención directa en el aula con los niños y las niñas.

2 Solo en casos puntuales, las observaciones continuaron en días señalados con el fin de recabar información complementaria. 
El trabajo en el aula universitaria se ha orientado a través de un nutrido grupo de estrategias de aula: exposiciones teóricas, debates o preparación en grupos de trabajo de materiales plásticos para el desarrollo de los talleres didácticos, entre otras. Las dos principales estrategias dirigidas a favorecer el trabajo cooperativo el aula y el desarrollo de competencias comunicativas en el alumnado han sido las asambleas de clase y el foro del campus virtual.

- Las asambleas. Una vez a la semana se efectuaba una asamblea en clase. El mobiliario del aula se desplazaba, distribuyendo las mesas y las sillas a lo largo de tres de los cuatro lados del espacio. La pizarra, el panel de proyección y la mesa docente ocupaban el cuarto lado del aula, su frontal. Esta disposición facilitaba el encuentro cara a cara de los alumnos y alumnas y contribuía a iniciar y sostener el debate en la clase. Cada semana, una pareja tomaba notas de los temas tratados durante la asamblea. Las actas resultantes era subidas a la plataforma del campus virtual de forma que los acuerdos adoptados y las decisiones asumidas en clase fueran accesibles a todo el alumnado.

- Construimos junt@s nuestros talleres. En el campus virtual de la asignatura se habilitó un foro de debate en el que tanto la docente como el alumnado podían iniciar hilos de conversación, ofreciendo aportes de variada naturaleza: imágenes y fotografías, propuestas de actividades y talleres, apuntes sobre la asamblea en el aula infantil, etc. Estos aportes no solo eran útiles para la planificación de los talleres del propio grupo de trabajo, sino también para el resto de compañeros y compañeras que accedían a los hilos de debate del foro.

\subsection{Fase tercera: la ejecución de los talleres didácticos}

El planteamiento de un taller didáctico por parte de alumnado universitario del primer curso del Grado en Educación Infantil constituye una labor compleja. El hecho de situarse la asignatura en el primer semestre de la planificación implica, además, una dificultad añadida por el desconocimiento generalizado del alumnado acerca de la educación infantil y su didáctica. Solo aquellos y aquellas estudiantes que disponen del título de Técnico Superior en Educación Infantil cuentan con ciertas herramientas formativas para el desempeño de los talleres. Por ese motivo, se decidió que la estructura y la dinámica de las asambleas serían las mismas en todas las aulas de educación infantil y en el salón de psicomotricidad reservado para el taller intergeneracional. Se acordó que los recursos a utilizar durante las asambleas serían la canción infantil Pequeño planeta y una lámina con el 
dibujo de la Tierra sonriente. Estos recursos materiales serían trabajados en cada aula de la escuela infantil durante las semanas previas a la ejecución de los talleres didácticos, de forma que, llegado el momento de las asambleas durante la semana por los derechos de la infancia, los niños y las niñas estuviesen plenamente familiarizados con estos materiales. En cuanto al diseño y a la creación de los talleres didácticos, más adelante se reserva un espacio a su descripción y análisis.

Es importante matizar que el inicio del diseño de los talleres didácticos fue simultáneo a las sesiones de observación en el aula y a la exposición y el estudio de los derechos de la infancia durante las clases teóricas de la asignatura. Esto facilitó que el alumnado universitario se apercibiese de la necesidad de conocer las características, las necesidades y los aprendizajes previos del grupo-clase de educación infantil para acometer el diseño de una intervención educativa. Las citas siguientes reflejan la colisión que puede producirse entre el diseño de una actuación educativa descontextualizada y la realidad escolar.

La primera de estas citas está tomada del correo electrónico del campus virtual y recoge el caso de la propuesta de un taller del que el alumnado reconoce su inviabilidad tras una conversación mantenida con la educadora del aula.

Buenos días [...], cuando fuimos ayer a la escuela infantil, teníamos pensado realizar con los pequeños talleres dedicados a la música y al baile. Habíamos pensado en cantar y bailar con ellos la canción llamada "el patio de mi casa", entre ellas.

Pero cuando llegamos le preguntamos a [...], la profesora de esa clase, que [sic] le parecía. Ella nos comento [sic] que era una buena idea pero no todos los pequeño caminan, por tanto, sería complicado bailar con todos. Por otro lado nos comento [sic] también que tienen hora de descanso y es probable que cuando vayamos muchos de ellos estén descansando.

Quedamos un poco desconcertadas y ahora no sabemos si seguir o no con ese taller [sic]

Gracias

Un cordial saludo. (Correo electrónico enviado por la alumna "A", el 21/20/2017)

La segunda cita, por su parte, se corresponde con el comentario escrito en uno de los foros habilitados en el campus virtual de la asignatura y documenta la decisión de diseñar un taller partiendo de los aprendizajes adquiridos en el aula de infantil por los niños y las niñas. 
El otro día junto con mis dos compañeras [...] estuvimos preguntándole a la profesora de un año las actividades que los niños eran capaces de realizar. Entre otras actividades, nos comentó que sabían cortar papeles con las manos y pegarlos. Hemos pensado que sería buena idea hacerlo y con los papeles cortados formar un árbol, relacionado con el tema del cambio climático. (Mensaje enviado al foro del Campus Virtual por la alumna "B", el 22/10/2017)

En la tabla siguiente se recogen las aulas y los talleres finalmente diseñados.

TABLA 1.

Aulas y talleres didácticos

\begin{tabular}{|l|l|l|}
\hline \multicolumn{3}{|c|}{ Talleres didácticos. El cambio climático en Educación infantil } \\
\hline & Lunes 20 de noviembre & Martes 21 de noviembre \\
\hline 1 AÑO A & Mis manos siembran verde (I) & La ruleta del tiempo \\
\hline 1 AÑO B Y BEBÉS & La Tierra me narra una historia & La Tierra me narra una historia \\
\hline 2 AÑOS A & Mis manos siembra verde (II) & Con mis sentidos conozco mi mundo \\
\hline 2 AÑOS B & Mis manos siembran verde (III) & El mar: un hogar limpio para los peces \\
\hline 2 AÑOS C & iYo actúo por el planeta! & Queremos un planeta feliz \\
\hline
\end{tabular}

- Queremos un planeta feliz: El taller parte de la creación por parte del alumnado universitario de dos paneles que representan la imagen de un planeta feliz y de un planeta triste. Con la ayuda del adulto, el alumnado de educación infantil identifica en láminas adhesivas qué acciones cotidianas suyas contribuyen a la construcción de un planeta feliz (regar una maceta, tirar residuos a la papelera, etc.) y las coloca en el panel correspondiente. Del mismo modo, el alumnado ha de Identificar con la ayuda del adulto y mediante el uso de láminas adhesivas, qué acciones repercuten en un planeta triste y situarlas en su panel.

- La Tierra me narra una historia: Relato del alumnado universitario de un cuento escenificado en el aula infantil. La narración se vale del soporte visual (imágenes, figuras y marionetas) y auditivo (batería de sonidos de la naturaleza)

- Con mis sentidos conozco mi mundo: Se trata de un taller intergeneracional en el que participan conjuntamente el alumnado y sus abuelos y abuelas. Bajo la dirección del estudiantado universitario, el alumnado de la escuela infantil ofrece a sus abuelos y abuelas materiales y productos de la naturaleza de texturas, sabores y olores diversos (por ejemplo: unas piedras, una hoja de hierbabuena, etc.). Finalmente, se seleccionan objetos de 
texturas diferentes y se introducen en globos, al tiempo que se facilita la experimentación con el sonido.

- La ruleta del tiempo: El estudiantado universitario elabora un mural con diversos elementos meteorológicos (el sol, la lluvia, la nieve y el viento). El alumnado de educación infantil trabaja estos elementos con materiales plásticos: algodón, pinturas, adhesivos y papel de colores. El orden de intervención sobre los elementos meteorológicos se establece mediante el uso de una ruleta.

- Mis manos siembran verde (I) y (II): Organizados en pequeños grupos de trabajo y con la colaboración del alumnado universitario, los niños y las niñas decoran tres macetas que sembrarán para permanecer en el aula a lo largo del curso.

- Mis manos siembran verde (I): Taller intergeneracional en el que participan los niños y las niñas de infantil y sus abuelos y abuelas. Se trata de realizar la siembra de una planta en macetas decoradas conjuntamente.

- El mar: un hogar limpio para los peces: Los niños y las niñas deben extraer peces de un recipiente que representa un mar contaminado y depositarlos en un recipiente idéntico al anterior aunque limpio y despejado. Cada pez se identifica con un color para reforzar el aprendizaje de los colores en los niños-as.

- ¡Yo actúo por el planeta! El estudiantado universitario realiza un mural que representa un entorno conocido por el alumnado de educación infantil (una playa, un parque, el patio de recreo, etc). En el mural se incluyen elementos contaminantes postizos, fácilmente desprendibles del mural y elaborados en cartón o cartulina (una lata, una botella, un papel, etc.). Los niños y las deben identificar los elementos distorsionantes del entorno, retirarlos y depositarlos en la papelera del aula.

\subsection{Fase cuarta: la valoración del proyecto Una semana por los derechos de la infancia. Algunas consideraciones finales}

La semana previa a la ejecución de los talleres didácticos en la escuela infantil Triquitraque se solicitó al estudiantado de educación superior una valoración del proyecto efectuado, así como del enfoque ApS en el aula universitaria. Con esta medida se pretendía que los alumnos y las alumnas ofreciesen una evaluación del proceso de enseñanza y aprendizaje seguido en las aulas desde el comienzo del curso académico. En las narraciones valorativas realizadas por el alumnado emerge un extenso abanico de reportes 
positivos derivados de su primera experiencia de ApS. De las producciones del estudiantado se han extraído las conclusiones que se detallan en la tabla siguiente

\section{TABLA 2}

\section{Aportes del ApS en los relatos narrativos del alumnado}

\begin{tabular}{|c|c|}
\hline \multicolumn{2}{|r|}{ La experiencia ApS en el aula universitaria } \\
\hline Aportaciones del ApS & Extractos de narraciones del alumnado \\
\hline $\begin{array}{l}\text { Refuerza el trabajo coo- } \\
\text { perativo entre el alumna- } \\
\text { do de educación superior. }\end{array}$ & $\begin{array}{l}\text { “[El ApS] favorece la cooperación y coordinación del grupo haciendo } \\
\text { que nos conozcamos más" (Estudiante 7) } \\
\text { "Me parece una dinámica muy acertada. Trabajamos en nuestro } \\
\text { propio grupo y además ayudamos a aquellos compañeros que lo } \\
\text { necesitan" (Estudiante 15) }\end{array}$ \\
\hline $\begin{array}{l}\text { Facilita las interacciones } \\
\text { entre el alumnado y la } \\
\text { cohesión del grupo-clase. }\end{array}$ & $\begin{array}{l}\text { "Esta forma de trabajar es muy buena [...] [te hace] interaccionar } \\
\text { con los compañeros" (Estudiante 1) } \\
\text { "Esta forma de trabajar en grupo es muy eficaz y útil, porque de una } \\
\text { manera u otra se fomenta la coordinación entre todos los miembros } \\
\text { del grupo, las relaciones sociales y la cooperación" (Estudiante 28). }\end{array}$ \\
\hline $\begin{array}{l}\text { so de } \\
\text { dizaje }\end{array}$ & $\begin{array}{l}\text { "Esta forma de trabajar es muy buena, porque se te hace la clase } \\
\text { divertida" (Estudiante 1) } \\
\text { "Se nos hace mucho más ameno y divertido" (Estudiante 4) } \\
\text { "Me parece una buna idea porque las clases son más dinámicas } \\
\text { e interesantes y el aprendizaje es más significativo y eficaz [...]" } \\
\text { (Estudiante 39) }\end{array}$ \\
\hline $\begin{array}{l}\text { Implica una aproxima- } \\
\text { ción a la experiencia } \\
\text { laboral }\end{array}$ & $\begin{array}{l}\text { "Me parece una opción bastante buena, ya que podemos tratar con } \\
\text { niños" (Estudiante 2) } \\
\text { "En mi opinión esta forma de trabajar me parece muy buena, ya } \\
\text { que, desde el principio, estamos en contacto con los niños y es la } \\
\text { mejor forma para aprender, debido a que en un futuro ese va a ser } \\
\text { nuestro trabajo día tras día [...]" (Estudiante 33) }\end{array}$ \\
\hline $\begin{array}{l}\text { Favorece la proyección } \\
\text { y la transferencia de los } \\
\text { aprendizajes teóricos a } \\
\text { un contexto práctico real. }\end{array}$ & $\begin{array}{l}\text { "Pienso que al trabajar llevando la teoría a la práctica es bastante } \\
\text { beneficioso, ya que podemos observar como [sic] son el comporta- } \\
\text { miento de los pequeños y su manera de actuar frente a las activi- } \\
\text { dades que proponemos. De esta forma, podemos comprobar si la } \\
\text { teoría se puede llevar a la práctica tal y como la vemos, o debemos } \\
\text { estar preparados para saber enfrentarnos a diversos casos [...]" } \\
\text { (Estudiante 45) }\end{array}$ \\
\hline
\end{tabular}

El equipo educativo de la escuela infantil, por su parte, ha realizado también una valoración del proyecto a través del recurso narrativo. La experiencia de ApS ha redundado beneficiosamente en la comunidad educativa y ha abierto una brecha hacia la comunicación y la cooperación mutua entre las familias y la escuela, lográndose así cubrir una de las demandas de la escuela con las que se inició este proyecto desde el enfoque de ApS. La participación de los abuelos y de las abuelas en las dinámicas educativas durante esa semana en el salón de psicomotricidad les ha facilitado la inmersión en 
las diversas formas en las que los derechos de la infancia se proyectan en la vida organizativa del centro. Se les hizo entrega, además, de un díptico que sintetizaba los compromisos principales de la escuela infantil con los derechos de la infancia que aparecen reflejados en su proyecto educativo de centro. Asimismo, el equipo de educadores ha valorado positivamente los talleres planificados y desarrollados por el alumnado universitario, estimándolos como iniciativas educativas innovadoras. Por último, la implicación a fondo y la buena disposición hacia la iniciativa en la toma de decisiones de los alumnos y de las alumnas del Grado en Educación infantil han sido destacadas en las narraciones valorativas aportadas.

\begin{abstract}
"Ha sido una gran experiencia para los niños, las familias y para nosotros ha sido una forma de llevar a cabo actividades con nuestros niños novedosa [sic] y diferente" (Educador 1).

"Debido a las ganas y al entusiasmo que han prestado tanto a los pequeños como a nosotros, han hecho que el centro en general se llene de vida e ilusión. Como todos sabemos este trabajo es totalmente vocacional y en ocasiones la rutina amerma [sic] las ganas de trabajar con nuevos temas que a priori parecen complicados para los más pequeños y gracias a esa inyección de energía que nos han traído ellos, la chispa ha vuelto a brillar" (Educador 2).
\end{abstract}

IMAGEN 1

\section{Detalle de un taller didáctico}

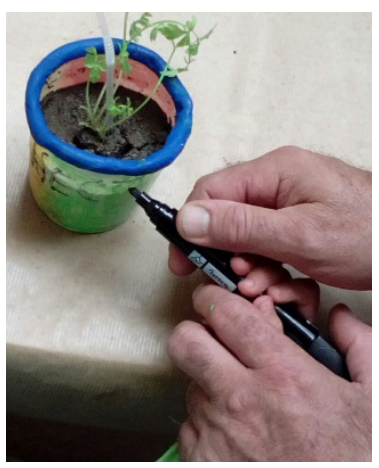

IMAGEN 2

\section{Desarrollo de un taller didáctico}

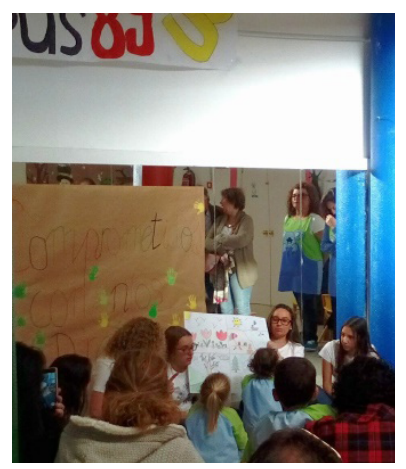

De igual modo, Unicef Comité-Andalucía se ha sumado a las felicitaciones al estudiantado universitario y a la comunidad educativa de la Enfantil Municipal Triquitraque por los resultados del proyecto Una semana por los derechos de la infancia. 


\section{IMAGEN 3}

\section{Tweet de Unicef-Comité Andalucía}

UNICEF C. Andalucía

@UNICEFAndalucia

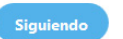

Los alumnos de @univcadiz han desarrollado

talleres en la escuela infantil El Triquitraque.

Comprometidos con la Educación en

Derechos de los más pequeños ¡Gracias por

vuestro trabajo! @campus_UNICEF

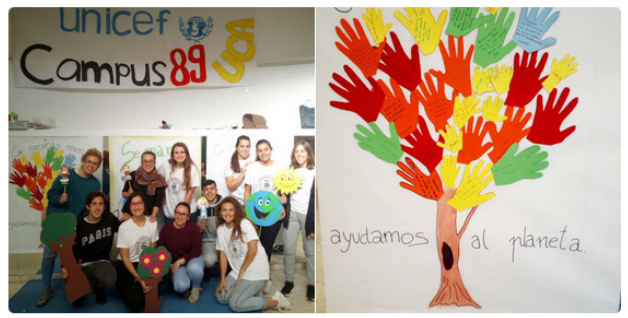

2:25 - 24 nov. 2017

Por último, los objetivos que se planearon abordar de cara a la asignatura Procesos Educativos en Educación Infantil se han alcanzado satisfactoriamente. En primer lugar y tal y como percibió el alumnado y reflejó en sus relatos narrativos, se ha favorecido la transferencia y la proyección de los contenidos teóricos relacionados con la didáctica de la educación infantil a un contexto sociocultural y educativo real y se ha facilitado, así, la consolidación de un aprendizaje significativo. En segundo lugar, la experiencia ApS ha permitido dirigir unos primeros pasos hacia el compromiso ético y la responsabilidad cívica como elementos constituyentes de la formación inicial de los educadores y educadoras infantiles. La implicación en proyectos de transformación social se configura así como una apuesta a largo plazo por un modelo de docente reflexivo y crítico.

\section{REFERENCIAS BIBLIOGRÁFICAS}

Batlle, R. (2011). ¿De qué hablamos cuando hablamos de aprendizaje-servicio?. Revista crítica, (972), 1-6. Recuperado de: http://roserbatlle.net/wp-content/uploads/2011/04/ de-que-hablamos-cuando-hablamos-de-aps.pdf [Fecha de acceso 29/11/2017]

Benítez Gavira, R.; Sánchez Calleja, L.; Zazuela, A. y García García, M. (2017). ¿Cómo diseñar proyectos de Aprendizaje y Servicio? Recomendaciones desde la práctica. En VV.AA. Libro de Actas de las II Jornadas de Innovación Docente UCA. Cádiz, 4-6 de Julio de 2017, pp. 186-190. Recuperado de: http://jornadas-innovaciondocente.uca.es/ wp-content/uploads/2017/02/libroActasJID2017.pdf [Fecha de acceso 29/11/2017]

Bisquerra, R. (2008). Educación para la ciudadanía y convivencia. El enfoque de la educación emocional. Bilbao: Wolters Kluwer. 
Bolívar, A. (2005). El lugar de la ética profesional en la formación universitaria. Revista Mexicana de Investigación Educativa, 10(24), 93-123.

Buxarrais, M. R.; Esteban, F.; Mellen, T. (2015). The state of ethical learning in the Spanish University System: Considerations for the European Higher Education Area. Higher Education Research and Development, 34(4), 472-485.

Consejería de Educación. Junta de Andalucía (2008). Decreto 428/2008, de 29 de julio, por el que se establece la ordenación y las enseñanzas correspondientes a la Educación Infantil en Andalucía. Recuperado de: http://www.juntadeandalucia.es/boja/2008/164/2 [fecha de acceso: 29/11/2017].

Esteban, F. y Buxarrais, M. R. (2004). El aprendizaje ético y la formación universitaria: más allá de la casualidad. Teoría de la Educación, (14), 91-108.

Esteban, F. y Martínez, M. (2012). ¿Son universidades todas las universidades? La universidad como comunidad ética. Bordón, 64(3), 77-92.

Francisco, A. y Moliner, L. (2010). El Aprendizaje-Servicio en la Universidad: una estrategia en la formación de ciudadanía crítica. Revista Electrónica Interuniversitaria de Formación del Profesorado, 13 (4), 69-77.

García García, M. y Cotrina García, M. J. (2015). El aprendizaje y servicio en la formación inicial del profesorado: de las prácticas educativas críticas a la institucionalización curricular. Profesorado. Revista de currículum y formación del profesorado, 19(1), 8-24.

Giroux, H. A. (1990). Los profesores como intelectuales. Hacia una pedagogía crítica del aprendizaje. Barcelona: Paidós-Madrid/MEC.

La Carta de la Tierra (2000). Recuperado de: http://earthcharter.org/invent/images/uploads/ echarter_spanish.pdf [fecha de acceso: 29/11/2017].

Martínez, M., Buxarrais, M. R. y Esteban, F. (2002). La universidad como espacio de aprendizaje ético. Revista Iberoamericana de Educación, 29, 17-28. Recuperado de https:// rieoei.org/RIE/article/view/949

Martínez, M. (2006). Formación para la ciudadanía y educación superior. Revista Iberoamericana de Educación, 42, 85-102. Recuperado a partir de https://rieoei.org/RIE/issue/view/60

Martínez, M. (2010). Aprendizaje servicio y construcción de ciudadanía activa en la universidad: la dimensión social y cívica de los aprendizajes académicos. En Miquel Martínez (Ed.), Aprendizaje servicio y responsabilidad social de las universidades (pp. 11 26). Barcelona: Octaedro.

Martínez-Odría, A. (2007). Service-learning o Aprendizaje-servicio. La apertura de la escuela a la comunidad local como propuesta de educación para la ciudadanía. Bordón, 59 (4), 627-640.

Mendia, R. (2009). Aprendizaje y servicio solidario, una propuesta educativa para el desarrollo de la competencia ciudadana. Revista del Forum Europeo de Administración Educativa, 86 (2), 1-6. Recuperado de: http://www.zerbikas.es/wp-content/ uploads/2015/09/0405MENapr.pdf [Fecha de acceso 29/11/2017]

Naval, C. (2010). Universidad y conciencia cívica. Algunas experiencias fructíferas: service learning y campus compact. En Miquel Martínez (Ed.), Aprendizaje servicio y responsabilidad social de las universidades (pp. 57-80). Barcelona: Octaedro.

Puig, J.M.; Batlle, R.; Bosch, C. y Palos, J. (2007). Aprendizaje servicio. Educar para la ciudadanía. Barcelona: Octaedro. 
Puig, J.M.; Gijón, M.; Martín, X. y Rubio, L. (2011). Aprendizaje-servicio y Educación para la Ciudadanía. Revista de Educación, (n.e.), 45-67.

Repáraz, C., Arbués, E., Naval, C. y Ugarte, C. (2015). El Índice Cívico de los universitarios. Sus conocimientos, actitudes y habilidades de participación social. Revista española de pedagogía, 73 (260), 23-51.

Rodríguez Gallego, M. R. (2014). El Aprendizaje-Servicio como estrategia metodológica de la Universidad. Revista Complutense de Educación, 25 (1), 95-113.

Schön, D. (1987). El profesional reflexivo: cómo piensan los profesionales cuando actúan. Barcelona: Paidós Iberia.

Tapia, M. N. (2010). Calidad académica y responsabilidad social: el aprendizaje servicio como puente entre dos culturas universitarias. En Miquel Martínez (Ed.), Aprendizaje servicio y responsabilidad social de las universidades (pp. 27-56). Barcelona: Octaedro.

UNESCO (2009). Conferencia Mundial sobre la Educación Superior. La nueva dinámica de la educación superior y la investigación para el cambio social y el desarrollo. París. Recuperado de: http://www.unesco.org/education/WCHE2009/comunicado_es.pdf. [Fecha de acceso 11/11/2017] 
